\title{
ON THE EXISTENCE OF UNITARY FLAT CONNECTIONS OVER THE PUNCTURED SPHERE WITH GIVEN LOCAL MONODROMY AROUND THE PUNCTURES*
}

\author{
INDRANIL BISWAS ${ }^{\dagger}$
}

1. Introduction. Let $X$ be the complement of a finite subset of the projective line $\mathbb{C P}^{1}$, or equivalently, it is a connected smooth affine curve of genus zero over $\mathbb{C}$. To each point $s \in \mathbb{C P}^{1}-X$ of the finite set we associate an orbit $C_{s}$ of the conjugation action of the unitary group $U(r)$ on itself. A natural class of examples of this situation is obtained by considering the local monodromy of a unitary local system over $X$. Our aim here is to characterize the examples that arise this way. More precisely, we give a sufficient condition on the collection $\left\{C_{s}\right\}$ for it to realize as the local monodromy of a unitary local system over $X$ [Theorem 3.10]. The condition is in the form of a finite set of inequalities constructed using the eigenvalues of the conjugacy classes $C_{s}$ and their multiplicity. For each such inequality, we give a condition which determines whether the validity of this inequality is necessary to ensure the existence of a unitary flat connection with the given local monodromy $\left\{C_{s}\right\}$ [Theorem 3.23].

A further restricted class of examples of such data $\left\{C_{s}\right\}$ is obtained by considering the local monodromy of irreducible unitary local systems over $X$. We give a similar condition on $\left\{C_{s}\right\}$ for it to realize as the local monodromy of an irreducible unitary local system over $X$.

These results were earlier proved for the special case of $U(2)$ [Bi].

There is a natural bijective correspondence between the set of all equivalence classes of irreducible representation of the fundamental group $\pi_{1}(X)$ into $U(r)$ and the set of all isomorphism classes of rank $r$ parabolic stable bundles over $\mathbb{C P}^{1}$ of parabolic degree zero and $\mathbb{C P}^{1}-X$ as the parabolic divisor. Furthermore, the space of equivalence classes of representations of $\pi_{1}(X)$ into $U(r)$ are in one-to-one correspondence with the space of $S$-equivalence classes of rank $r$ parabolic semistable bundles of parabolic degree zero [Si1], [MS]. In these correspondences, fixing the conjugacy class of the local monodromy around $s \in \mathbb{C P}^{1}-X$ is equivalent to fixing the parabolic data at $s$.

Our approach here is to try to obtain necessary and sufficient conditions for the existence of a parabolic (semi)stable bundle with a given parabolic data.

In $[\mathrm{FS}]$ and $[\mathrm{N}]$ the cohomology groups of a smooth moduli space of parabolic stable bundles have been computed. One possible way of concluding that a given space is nonempty is to check that its 0 -th cohomology is nonzero. However, the computations in [FS] and [N] are made under the assumption that the moduli space is nonempty, thus making them unsuitable for the problem addressed here.

If $\left\{\Gamma_{s}\right\}_{s \in \mathbb{C P}^{1}-X}$ are conjugacy classes in $S L(r, \mathbb{C})$ with at-least one conjugacy class $\Gamma_{s}$ having distinct eigenvalues, then a theorem of Simpson gives a necessary and sufficient condition on $\left\{\Gamma_{s}\right\}$ for it to realize as the local monodromy of a $S L(r, \mathbb{C})$ local system over $X$ [Si2]. However, as already noted in [Bi], the solutions for $S L(r, \mathbb{C})$ and $U(r)$ are quite different. What goes into the condition for the existence of a $S L(r, \mathbb{C})$ local system with given local monodromy, is the multiplicity of the eigenvalues of the

${ }^{*}$ Received July 21, 1998; accepted for publication September 25, 1998.

†School of Mathematics, Tata Institute of Fundamental Research, Homi Bhabha Road, Bombay 400005, INDIA (indranil@math.tifr.res.in). 
given conjugacy classes. In contrast - in the case of $U(r)$, the actual eigenvalues, and not just their multiplicity, feature in the inequalities in Theorems 3.10 and 3.23.

Thanks are due to C. S. Seshadri for posing the question addressed here.

2. Preliminaries. Let

$$
S:=\left\{s_{1}, \ldots, s_{d}\right\} \subset \mathbb{C P}^{1}
$$

be a finite set of points of the complex projective line. We shall recall the definition of a parabolic bundle over $\mathbb{C P}^{1}$ with $S$ as the parabolic points [MS, Definition 1.5].

Definition 2.1. A parabolic bundle over $\mathbb{C P}^{1}$, with $S$ as the parabolic divisor, consists of the following:

1. A rank $r$ holomorphic vector bundle $E$ over $\mathbb{C P}^{1}$;

2. for every $s \in S$, a filtration by subspaces, of the fiber $E_{s}$ of $E$ at $s$,

$$
E_{s}=F^{1} E_{s} \supset F^{2} E_{s} \supset \ldots \supset F^{l_{s}-1} E_{s}^{\prime} \supset F^{l_{s}} E_{s} \supset F^{l_{s}+1} E_{s}=0
$$

called the quasi-parabolic flag;

3. for every $s \in S$, a string of real numbers $\left\{\bar{\alpha}_{i}^{s}\right\}_{1 \leq i \leq l_{s}}$, called the parabolic weights, such that

$$
0 \leq \bar{\alpha}_{1}^{s}<\bar{\alpha}_{2}^{s}<\ldots<\bar{\alpha}_{l_{s}-1}^{s}<\bar{\alpha}_{l_{s}}^{s}<1
$$

the weight $\bar{\alpha}_{i}^{s}$ corresponds to the subspace $F^{i} E_{s}$ in the quasi-parabolic flag.

We shall denote the parabolic bundle, defined above, by $E_{*}$.

The dimension of $F^{i} E_{s} / F^{i+1} E_{s}$ is called the multiplicity of the weight $\bar{\alpha}_{i}^{s}$; this multiplicity will be denoted by $m_{s}^{i}$. 1.11]:

The parabolic degree of $E_{*}$, denoted by par-deg $E_{*}$, is defined as [MS, Definition

$$
\operatorname{par}-\operatorname{deg} E_{*}:=\operatorname{degree} E+\sum_{s \in S} \sum_{i=1}^{l_{s}} m_{s}^{i} \bar{\alpha}_{i}^{s}
$$

For any $s \in S$ and any integer $j \in[1, r]$, define $k(s, j)$ to be the integer in the interval $\left[1, l_{s}\right]$ which is the minimum of all $t \in\left[1, l_{s}\right]$ satisfying the following condition:

$$
j \leq \sum_{i=1}^{t} m_{s}^{i}
$$

Note that the inequality $j \geq k(s, j)$ is valid.

For any $s \in S$ and $j \in[1, r]$, define

$$
\alpha_{j}^{s}:=\bar{\alpha}_{k(s, j)}^{s}
$$

where $k(s, j)$ is defined in (2.3). Thus we have the following string of numbers:

$$
0 \leq \alpha_{1}^{s} \leq \alpha_{2}^{s} \leq \ldots \leq \alpha_{r}^{s}<1
$$

The type of the flag $\left\{F^{i} E_{s}\right\}$ in Definition 2.1(2) (that is the integers $m_{s}^{i}$ ) can be read off from the sequence $\left\{\alpha_{i}^{s}\right\}$ by just counting the number of times each $\alpha_{i}^{s}$ repeats. 
The parabolic degree of $E_{*}$, defined in (2.2), now becomes

$$
\text { par-deg } E_{*}:=\operatorname{degree} E+\sum_{s \in S} \sum_{i=1}^{r} \alpha_{i}^{s}
$$

The quotient par- $\operatorname{deg} E_{*} / \operatorname{rank} E$ is called the slope of $E_{*}$, and it is denoted by $\operatorname{par}-\mu\left(E_{*}\right)$.

For a parabolic bundle $E_{*}$, any subbundle of $E$ has an induced parabolic structure induced by $E_{*}$. If $V$ is a subbundle of $E$, then the quasi-parabolic flag in $V_{s}$ is simply the intersection of $V_{s}$ with the flag in Definition 2.1(2). If $V_{s} \cap F^{i+1} E_{s}$ is a proper subspace of $V_{s} \cap F^{i} E_{s}$, then the parabolic weight of $V_{s} \cap F^{i} E_{s}$ is $\bar{\alpha}_{i}^{s}$. Let $V_{*}$ denote the parabolic bundle obtained this way.

The parabolic bundle $E_{*}$ is called parabolic stable (respectively, parabolic semistable) if for every nonzero subbundle $V \neq E$, the following inequality between the slopes of $E_{*}$ and $V_{*}$ is valid [MS, Definition 1.13]:

$$
\operatorname{par}-\mu\left(V_{*}\right)<\operatorname{par}-\mu\left(E_{*}\right)\left(\text { respectively, } \quad \operatorname{par}-\mu\left(V_{*}\right) \leq \operatorname{par}-\mu\left(E_{*}\right)\right)
$$

A parabolic semistable bundle is called parabolic polystable if it is a direct sum of parabolic stable bundles of same slope.

In [Si1] and [MS] a bijective correspondence between

$$
\operatorname{Hom}\left(\pi_{1}\left(\mathbb{C P}^{1}-S\right), U(r)\right) / U(r),
$$

namely the space of equivalence classes of $U(r)$ representations of the fundamental group of $\mathbb{C P}^{1}-S$, and the space of all parabolic polystable bundles (or equivalently, $S$-equivalence classes of parabolic semistable bundles) over $\mathbb{C P}^{1}$ of parabolic degree zero and with $S$ as the parabolic points, has been established. In this correspondence, the subspace $\operatorname{Hom}^{\operatorname{irr}}\left(\pi_{1}\left(\mathbb{C P}^{1}-S\right), U(r)\right) / U(r)$, consisting of all irreducible representations, corresponds to the space of all parabolic stable bundles of parabolic degree zero. For a parabolic polystable bundle $E_{*}$ of parabolic degree zero and with parabolic structure as in Definition 2.1, the corresponding equivalence class of representations $\rho \in \operatorname{Hom}\left(\pi_{1}\left(\mathbb{C P}^{1}-S\right), U(r)\right) / U(r)$ has the property that the local monodromy for $\rho$ around any $s \in S$ has $\left\{\exp \left(2 \pi \sqrt{-1} \alpha_{1}^{s}\right), \ldots, \exp \left(2 \pi \sqrt{-1} \alpha_{r}^{s}\right)\right\}$ as the eigenvalues, where $\alpha_{i}^{s}$ are defined in (2.4). Thus the parabolic data at a parabolic point determines - and is determined by - the conjugacy class of the local monodromy of the corresponding unitary representation.

For a parabolic bundle, say $E_{*}$, of parabolic degree zero, $\sum_{s \in S} \sum_{i=1}^{r} \alpha_{i}^{s}$ must be an integer, since it is equal to $-\operatorname{deg} E$. Since the product of the monodromies around all the punctures is the identity element, for any $\rho \in \operatorname{Hom}\left(\pi_{1}\left(\mathbb{C P}^{1}-S\right), U(r)\right) / U(r)$, considering the determinant of this product, the condition $\sum_{s \in S} \sum_{i=1}^{r} \alpha_{i}^{s} \in \mathbb{N}$ is obtained again.

The following condition for the existence of a representation of $\pi_{1}\left(\mathbb{C P}^{1}-S\right)$ in $U(2)$ is known.

Theorem $2.7[\mathrm{Bi}]$. Assume that the integer $\sum_{s \in S}\left(\alpha_{1}^{s}+\alpha_{2}^{s}\right)$ is odd (respectively, even), say $2 N+1$ (respectively, $2 N$ ). Then there is a parabolic stable bundle of parabolic degree zero and with parabolic weights $\left\{\alpha_{1}^{s}, \alpha_{2}^{s}\right\}$ at $s \in S$, if and only if for every subset $D \subseteq S$ of cardinality $2 j$ (respectively, $2 j+1$ ), where $j$ is a nonnegative integer, the following inequality holds:

$$
-N-j+\sum_{s \in D} \alpha_{2}^{s}+\sum_{s \in S-D} \alpha_{1}^{s}<0
$$


There is a parabolic semistable bundle with the given parabolic data if and only if the left-hand side of the above inequality is nonnegative.

Generalizing Theorem 2.7, in the next section we shall give a criterion for the existence of a parabolic (semi)stable bundle over $\mathbb{C P}^{1}$ with a given parabolic data.

3. A criterion for the existence of a parabolic stable bundle. Fix a parabolic data for a parabolic bundle of rank $r$ and of parabolic degree zero, with $S$ as the parabolic points. This simply means that for each $s \in S$, we have a string of numbers $\left\{\alpha_{i}^{s}\right\}$, where $1 \leq i \leq r$, satisfying the condition (2.5), and $\sum_{s \in S} \sum_{i=1}^{r} \alpha_{i}^{s}$ is an integer. Let $M \geq 0$ and $m \in[0, r-1]$ be the integers such that

$$
\sum_{s \in S} \sum_{i=1}^{r} \alpha_{i}^{s}=M r-m
$$

For any $s \in S$, define $l_{s}$ to be the number of distinct $\alpha_{i}^{s}$. The multiplicity of the $i$-th one, in the increasing order of $\left\{\alpha_{s}^{j}\right\}$, is denoted by $m_{s}^{i}$.

Let $G(s, r)$ denote the flag variety consisting of all flags of the type

$$
\mathbb{C}^{r}=V^{1} \supset V^{2} \supset \ldots \supset V^{l_{s}-1} \supset V^{l_{s}} \supset V^{l_{s}+1}=0
$$

in $\mathbb{C}^{r}$ such that $\operatorname{dim}\left(V^{i} / V^{i+1}\right)=m_{s}^{i}$. For any $n \in[1, r-1]$, fix a subspace $W_{n}$ on $\mathbb{C}^{r}$, say the subspace spanned by the first $n$ basis vectors.

For an integer $n \in[1, r-1]$, let $\mathcal{I}(s, n)$ denote the set of all maps

$$
\lambda:\left\{1,2 \ldots, l_{s}\right\} \rightarrow\{0,1,2, \ldots, n\}
$$

such that $\sum_{i=1}^{l_{s}} \lambda(i)=n$ and $\lambda(i) \leq m_{s}^{i}$.

For any $\lambda \in \mathcal{I}(s, n)$, define

$$
S\left(W_{n}, \lambda\right) \subseteq G(s, r)
$$

to be the Schubert variety consisting of all flags of the type (3.2) such that

$$
\operatorname{dim}\left(W_{n} \cap V^{i}\right) \geq \sum_{j=i}^{l_{s}} \lambda(j)
$$

Clearly $S\left(W_{n}, \lambda\right)$ is nonempty. It is known that $S\left(W_{n}, \lambda\right)$ is an irreducible subvariety of $G(s, r)$. Now define

$$
\zeta(s, \lambda):=\operatorname{codim} S\left(W_{n}, \lambda\right)
$$

to be the codimension of $S\left(W_{n}, \lambda\right)$ in $G(s, r)$.

For any $\lambda \in \mathcal{I}(s, n)$, as above, define

$$
\omega(s, \lambda):=\sum_{i=1}^{l_{s}} \lambda(i) \alpha_{i}^{s}
$$

Let $\mathcal{I}(n)$ denote the Cartesian product of $\mathcal{I}(s, n)$. In other words,

$$
\mathcal{I}(n):=\left\{\prod_{s \in S} \lambda_{s} \mid \lambda_{s} \in \mathcal{I}(s, n)\right\}
$$


Now, for any $\bar{\lambda}=\prod_{s \in S} \lambda_{s} \in \mathcal{I}(n)$ define

$$
\zeta(\bar{\lambda}):=\sum_{s \in S} \zeta\left(s, \lambda_{s}\right)
$$

where $\zeta\left(s, \lambda_{s}\right)$ is defined in (3.5). Also define

$$
\omega(\bar{\lambda})=\sum_{s \in S} \omega\left(s, \lambda_{s}\right)
$$

where $\omega\left(s, \lambda_{s}\right)$ is defined in (3.6).

We need to make one more definition before stating a theorem.

For any integer $n \in[1, r-1]$, let $K(n)$ denote the set of all

$$
\bar{k}:=\left\{k_{1}, k_{2}, \ldots, k_{n}\right\} \in \mathbb{Z}^{n}
$$

with $k_{1} \leq k_{2} \leq \ldots \leq k_{n}$ and $k_{n}-k_{1} \leq 1$, such that all $k_{i} \geq-1$ and at-most $m$ of them are -1 , where $m$ is defined in (3.1). So either $k_{i}=k_{1}$ or $k_{i}=k_{1}+1$. Define the function

$$
\delta: K(n) \longrightarrow \mathbb{Z}
$$

by $\delta(\bar{k})=n(m+r)-n^{2}+r \sum_{i=1}^{n} k_{i}$. Note that $\delta(\bar{k})$ is a nonnegative integer. We shall see later that the number $\delta(\bar{k})$ has the following interpretation: denoting the line bundle of degree $i$ over $\mathbb{C P}^{1}$ by $\mathcal{O}(i)$, the number $\delta(\bar{k})$ coincides with the dimension of the moduli space of all rank $n$ subbundles of the vector bundle $\mathcal{O}^{\oplus(r-m)} \oplus \mathcal{O}(1)^{\oplus m}$ which decompose as $\bigoplus_{i=1}^{n} \mathcal{O}\left(-k_{i}\right)$.

Now we are in a position to state the criterion for the existence of a parabolic (semi)stable bundle of rank $r$ and parabolic degree zero, and with $\left\{\alpha_{i}^{s}\right\}$ as the parabolic weights at $s$.

Theorem 3.10. Let $n \in[1, r-1]$ be an integer, and $\bar{k}=\left\{k_{1}, \ldots k_{n}\right\} \in K(n)$, and $\bar{\lambda}=\prod_{s \in S} \lambda_{s} \in \mathcal{I}(n)$, with

$$
\zeta(\bar{\lambda}) \leq \delta(\bar{k})
$$

There is parabolic semistable bundle of rank $r$ and of parabolic degree zero over $\mathbb{C P}^{1}$, with $S$ as the set of parabolic points and $\left\{\alpha_{i}^{s}\right\}$ as the parabolic data at $s \in S$, if for every such pair $\bar{k}$ and $\bar{\lambda}$, the following inequality is valid:

$$
\omega(\bar{\lambda})-M n-\sum_{i=1}^{n} k_{i} \leq 0
$$

Moreover, there is a parabolic stable bundle, with the given parabolic data, if the lefthand side of the inequality (3.11) is strictly negative for every pair $\bar{k}$ and $\bar{\lambda}$ satisfying the above condition.

The functions $\zeta, \omega$ and $\delta$ were defined in (3.7), (3.8) and (3.9) respectively.

The number of nontrivial inequalities that appear in Theorem 3.10 is actually finite. Indeed, if, for example, $k_{n}>M r$, then the left-hand side of (3.11) is automatically strictly negative. 
Proof of Theorem 3.10. For any $j \in \mathbb{Z}$ let $\mathcal{O}(j)$ denote the line bundle over $\mathbb{C P}^{1}$ of degree $j$. Define

$$
E:=\mathcal{O}(-M)^{\oplus(r-m)} \bigoplus \mathcal{O}(-M+1)^{\oplus m}
$$

to be the vector bundle of $\operatorname{rank} r$ on $\mathbb{C P}^{1}$. The theorem will be proved by constructing a parabolic structure on $E$ of the given type which is also parabolic semistable. This will be done by first considering all parabolic structures on $E$ and then omitting all those which admit candidates for destabilizing subbundles. The inequality condition (3.11) will be used in ensuring that what remains is nonempty from dimension considerations.

Take any $\bar{k}:=\left\{k_{1}, \ldots, k_{n}\right\} \in K(n)$. Define

$$
V(\bar{k}):=\bigoplus_{i=1}^{n} \mathcal{O}\left(-M-k_{i}\right)
$$

to be the vector bundle of rank $n$ on $\mathbb{C P}^{1}$. The given condition

$$
\#\left\{i \mid k_{i}=-1\right\} \leq m
$$

(\# denotes the cardinality of a set) ensures that $V(\bar{k})$ can be realized as a subbundle of $E$. To see this, let $k_{i}=-1$ for $i \leq m^{\prime}$, and $k_{i} \geq 0$ for $i>m^{\prime}$. Let $L_{j}$ denote the $j$-th direct summand line bundle in the direct sum (3.12). In other words, $L_{j}=\mathcal{O}(-M)$ or $\mathcal{O}(-M+1)$ depending on whether $j \leq r-m$ or not. For any $i \leq m^{\prime}$, let $\bar{f}_{i}$ be the identity homomorphism from $\mathcal{O}\left(-M-k_{i}\right)$ to $L_{r-i+1}$. For any $i \in\left[m^{\prime}+1, n\right]$, fix a pair of nonzero homomorphisms $\left(f_{i, 1}, f_{i, 0}\right)$,

$$
\bar{f}_{i}:=\left(f_{i, 0}, f_{i, 1}\right): \mathcal{O}\left(-M-k_{i}\right) \longrightarrow L_{i-m^{\prime}} \oplus L_{i-m^{\prime}+1}
$$

such that the two divisors $\left\{z \mid f_{i, 0}(z)=0\right\}$ and $\left\{z \mid f_{i, 1}(z)=0\right\}$, and also the two divisors $\left\{z \mid f_{i, 1}(z)=0\right\}$ and $\left\{z \mid f_{i+1,0}(z)=0\right\}$ are disjoint. It is easy to check that the homomorphism

$$
\bigoplus \bar{f}_{i}: V(\bar{k}) \longrightarrow E
$$

is injective with its image being a subbundle of $E$, or equivalently, the quotient $E / V(\bar{k})$ is torsion-free.

Let $\mathcal{M}(\bar{k})$ denote the moduli space of all subbundles of $E$ isomorphic to $V(\bar{k})$. In other words, $\mathcal{M}(\bar{k})$ is the quotient, by the automorphism group $\operatorname{Aut}(V(\bar{k}))$, of the space all injective homomorphisms of $V(\bar{k})$ into $E$ with a torsion-free quotient. The space $\mathcal{M}(\bar{k})$ is nonempty by the previous remark.

For any $s \in S$, let $G\left(E_{s}\right)$ denote the flag variety, consisting of flags in $E_{s}$ of type

$$
E_{s}=E_{s}^{1} \supset E_{s}^{2} \supset \ldots \supset E_{s}^{l_{s}-1} \supset E_{s}^{l_{s}} \supset E_{s}^{l_{s}+1}=0
$$

such that $\operatorname{dim}\left(E_{s}^{i} / E_{s}^{i+1}\right)=m_{s}^{i}$. Evidently the Cartesian product

$$
\mathcal{G}:=\prod_{s \in S} G\left(E_{s}\right)
$$

parametrizes the space of all quasi-parabolic flags of the given type on $E$. We shall construct a family of Schubert varieties in $G\left(E_{s}\right)$ parametrized by $\mathcal{M}(\bar{k})$. 
Take any $\bar{\lambda}=\prod_{s \in S} \lambda_{s} \in \mathcal{I}(n)$. Imitating the definition of $S\left(W_{n}, \lambda\right)$ in (3.4), for any subbundle $F \subset E$ with $F \in \mathcal{M}(\bar{k})$, define the Schubert variety

$$
S\left(F, \lambda_{s}\right) \subseteq G\left(E_{s}\right)
$$

by replacing $\mathbb{C}^{r}, W_{n}$ and $\lambda$ in (3.4) by $E_{s}, F_{s}$ and $\lambda_{s}$ respectively. Define the subvariety

$$
S(F, \bar{\lambda}):=\prod_{s \in S} S\left(F, \lambda_{s}\right) \in \prod_{s \in S} G\left(E_{s}\right)=\mathcal{G}
$$

of $\mathcal{G}$ given by the Cartesian product of all $S\left(F, \lambda_{s}\right)$.

Let

$$
\mathcal{C}:=\{(F, G) \mid G \in S(F, \bar{\lambda})\} \subseteq \mathcal{M}(\bar{k}) \times \mathcal{G}
$$

be the incidence variety. Denoting the projection of $\mathcal{M}(\bar{k}) \times \mathcal{G}$ onto $\mathcal{G}$ by $p_{2}$, define

$$
\mathcal{H}(\bar{k}, \bar{\lambda}):=p_{2}(\mathcal{C})
$$

In other words, the equality

$$
\mathcal{H}(\bar{k}, \bar{\lambda})=\bigcup_{F \in \mathcal{M}(\bar{k})}\left(\prod_{s \in S} S\left(F, \lambda_{s}\right)\right) \subseteq \mathcal{G}
$$

is valid. Let $\overline{\mathcal{H}(\bar{k}, \bar{\lambda})}$ denote the Zariski closure of $\mathcal{H}(\bar{k}, \bar{\lambda})$ in $\mathcal{G}$.

Consider the union

$$
\Theta:=\bigcup_{n \in[1, r-1],\{\bar{\lambda} \in \mathcal{I}(n), \bar{k} \in K(n) \mid \zeta(\bar{\lambda})>\delta(\bar{k})\}} \overline{\mathcal{H}(\bar{k}, \bar{\lambda})}
$$

taken over all $n$ and all pairs $(\bar{k}, \bar{\lambda})$ with $\zeta(\bar{\lambda})>\delta(\bar{k})$.

The following proposition will be needed to estimate the dimension of $\mathcal{H}(\bar{k}, \bar{\lambda})$.

Proposition 3.18. The variety $\mathcal{M}(\bar{k})$ is of dimension $\delta(\bar{k})$ (defined in (3.9)).

Proof. We shall first show that $V(\bar{k})$ is rigid.

According to a theorem due to Grothendieck, every vector bundle over $\mathbb{C P P}^{1}$ decomposes as a direct sum of line bundles. It is easy to compute the dimension of the space of global endomorphisms of $\bigoplus_{i=1}^{N} \mathcal{O}\left(b_{i}\right)$, where $b_{1} \leq b_{2} \leq \ldots \leq b_{N}$, to be the following:

$$
\operatorname{dim} H^{0}\left(\mathbb{C P}^{1}, \operatorname{End}\left(\bigoplus_{i=1}^{N} \mathcal{O}\left(b_{i}\right)\right)\right)=\sum_{i \leq j}\left(b_{j}-b_{i}+1\right)+\sum_{i>j} \max \left\{b_{j}-b_{i}+1,0\right\}
$$

Now, for a fixed integer $N$ and fixed total degree $\sum_{i=1}^{N} b_{i}$, the right-hand side of (3.19) takes the minimum possible value, which is $N^{2}$, if and only if $b_{N}-b_{1} \leq 1$. Invoking semicontinuity, in a family of vector bundles over $\mathbb{C P}^{1}$ of rank $N$ and degree $\sum_{i=1}^{N} b_{i}$, with $\left|b_{i}-b_{j}\right| \leq 1$ for $1 \leq i, j \leq N$, the subvariety of the parameter space over which the vector bundle decomposes as $\bigoplus_{i=1}^{N} \mathcal{O}\left(b_{i}\right)$, is a Zariski open subset.

The tangent space of $\mathcal{M}(\bar{k})$ at any point $f \in \mathcal{M}(\bar{k})$ is:

$$
T_{f} \mathcal{M}(\bar{k}):=H^{0}\left(\mathbb{C P}^{1}, \operatorname{Hom}\left(V(\bar{k}), \frac{E}{V(\bar{k})}\right)\right)
$$


Indeed, this is immediate from the combination of the description of the tangent space of a Grassmannian together with the above observation that $V(\bar{k})$ is rigid.

Consider the following exact sequence of vector bundles:

$$
0 \longrightarrow \operatorname{End}(V(\bar{k}), V(\bar{k})) \longrightarrow \operatorname{Hom}(V(\bar{k}), E) \longrightarrow \operatorname{Hom}\left(V(\bar{k}), \frac{E}{V(\bar{k})}\right) \longrightarrow 0
$$

Since the vector bundle $\operatorname{Hom}(V(\bar{k}), E)$ is a direct sum of line bundles of degree atleast -1 , we have $H^{1}\left(\mathbb{C P P}^{1}, \operatorname{Hom}(V(\bar{k}), E)\right)=0$. Now the long exact sequence of cohomologies and the Riemann-Roch theorem give:

$$
\begin{gathered}
\operatorname{dim} H^{0}\left(\mathbb{C P}^{1}, \operatorname{Hom}\left(V(\bar{k}), \frac{E}{V(\bar{k})}\right)\right)=\operatorname{dim} H^{0}\left(\mathbb{C P}^{1}, \operatorname{Hom}(V(\bar{k}), E)\right) \\
+\operatorname{dim} H^{1}\left(\mathbb{C P}^{1}, \operatorname{End}(V(\bar{k}))\right)-\operatorname{dim} H^{0}\left(\mathbb{C P}^{1}, \operatorname{End}(V(\bar{k}))\right) \\
==n(m+r)+r \sum_{i=1}^{n} k_{i}-n^{2}=\delta(\bar{k})
\end{gathered}
$$

In other words, $\mathcal{M}(\bar{k})$ is a smooth variety of dimension $\delta(\bar{k})$. This completes the proof of the proposition. $\square$

From Proposition 3.18 the following inequality for the dimension of $\mathcal{H}(\bar{k}, \bar{\lambda})$ is obtained:

$$
\operatorname{dim} \mathcal{H}(\bar{k}, \bar{\lambda}) \leq \operatorname{dim} \mathcal{C}=\delta(\bar{k})+\operatorname{dim} \mathcal{G}-\zeta(\bar{\lambda})
$$

Therefore, $\operatorname{dim} \mathcal{H}(\bar{k}, \bar{\lambda})<\operatorname{dim} \mathcal{G}$ if $\zeta(\bar{\lambda})>\delta(\bar{k})$. Thus $\Theta$ is a countable union of subvarieties of $\mathcal{G}$ of dimensions strictly less than that of $\mathcal{G}$. (If $\operatorname{dim} \mathcal{G}=0$, then $\Theta$ is empty.) This immediately yields that the complement

$$
\mathcal{U}:=\mathcal{G}-\Theta
$$

is nonempty.

Recall that $\mathcal{G}$ parametrizes the space of parabolic structures of the given type on $E$. Evidently Theorem 3.10 is an immediate consequence of the following proposition:

Proposition 3.21. Assume that the inequality condition (3.11) is valid. Then the parabolic structure of $E$ corresponding to every point in $\mathcal{U}$ is actually parabolic semistable. Moreover, if the left-hand side of (3.11) is strictly negative, then every point of $\mathcal{U}$ represents a parabolic stable structure on $E$.

Proof of Proposition 3.21. Let $E_{*}$ denote a parabolic structure on $E$ corresponding to a point

$$
\bar{B}:=\prod_{s \in S} B_{s} \in \mathcal{U}
$$

where

$$
B_{s}:=\left\{E_{s}=E_{s}^{1} \supset E_{s}^{2} \supset \ldots \supset E_{s}^{l_{s}-1} \supset E_{s}^{l_{s}} \supset E_{s}^{l_{s}+1}=0\right\}
$$


is a flag in $E_{s}$ as in (3.14).

Let $F=V(\bar{k})$ be a subbundle of rank $n$ of $E$, where $\bar{k} \in K(n)$; the vector bundle $V(\bar{k})$ is defined in (3.13). Construct an element $\lambda_{n}$ of the set $\mathcal{I}(s, n)$ (defined in (3.3)) using the condition

$$
\sum_{j=i}^{l_{s}} \lambda_{s}(j)=\operatorname{dim}\left(E_{s}^{i} \cap F_{s}\right)
$$

where $E_{s}^{i}$ is defined in (3.22). Furthermore, define $\bar{\lambda}:=\prod_{s \in S} \lambda_{s}$.

Evidently the left-hand side of the inequality (3.11) is the parabolic degree of $F$ with the parabolic structure induced by $E_{*}$ (after substituting $\bar{\lambda}$ constructed above in (3.11)). Indeed, $\operatorname{deg} F=-M n-\sum_{i=1}^{n} k_{i}$, and the parabolic weight at $s \in S$ is $\omega\left(s, \lambda_{s}\right)$, where the function $\omega(s,-)$ is defined in (3.6).

Since $\bar{B} \in \mathcal{U}$, we have $\zeta(\bar{\lambda}) \leq \delta(\bar{k})$. So the inequality (3.11), combined with the above observation on the parabolic degree of $F$, implies that the subbundle $F$ does not violate the semistability condition for $E_{*}$.

Let

$$
F^{\prime}=\bigoplus_{i=1}^{n} \mathcal{O}\left(b_{i}\right)
$$

be a subbundle of $E$, with $b_{1} \leq b_{2} \leq \ldots \leq b_{n}$. The space of subbundles of $E$ of rank $n$ and of total degree $\sum_{i=1}^{n} b_{i}$ is parametrized by an irreducible variety; we shall call this variety as $\mathcal{N}$. Using semicontinuity for the dimension of the space of global endomorphisms and (3.19), we conclude that there is a unique $\bar{k} \in K(n)$ with the property that all the points in $\mathcal{N}$ such that the corresponding subbundle is isomorphic to $V(\bar{k})$, constitute a Zariski open dense subset of $\mathcal{N}$. Now we observe that the Zariski closures of $\mathcal{H}(\bar{k}, \bar{\lambda})$ are removed in the construction of $\mathcal{U}$ in (3.20). Repeating the earlier argument after substituting this new $\bar{k}$ we conclude that $F^{\prime}$ cannot violate the semistability condition for $E_{*}$.

The same argument yields the stability of $E_{*}$ if the left-hand side of (3.11) is strictly negative. This completes the proof of the proposition. $\square$

We already observed that Proposition 3.21 completes the proof of Theorem 3.10.

Note that the condition $\zeta(\bar{\lambda}) \leq \delta(\bar{k})$ was invoked in the proof Theorem 3.10 only to ensure that the dimension of the subvariety $\overline{\mathcal{H}(\bar{k}, \bar{\lambda})}$ is strictly less than the dimension of $\mathcal{G}$ whenever the condition fails. Define

$$
\mathcal{U}^{\prime}:=\mathcal{G}-\bigcup_{\mathcal{H}(\bar{k}, \bar{\lambda}) \neq \mathcal{G}} \overline{\mathcal{H}(\bar{k}, \bar{\lambda})}
$$

to be the complement in $\mathcal{G}$ of all $\overline{\mathcal{H}(\bar{k}, \bar{\lambda})}$ which are proper subvarieties. Then the proof of the Proposition 3.21 goes through without any change if we replace $\mathcal{U}$ (defined in (3.20)) by $\mathcal{U}^{\prime}$. In other words, what we have actually proved is apparently a bit stronger than Theorem 3.10. More precisely, we have actually proved the following: if the inequality (3.11) is valid for all pairs

$$
(\bar{k}, \bar{\lambda}) \in K(n) \times \mathcal{I}(n)
$$


with $\overline{\mathcal{H}(\bar{k}, \bar{\lambda})}=\mathcal{G}$, then there is a parabolic semistable bundle with the given parabolic data. Moreover, the existence of a parabolic stable bundle is ensured if the strict inequality is valid.

There are examples, with $r=4$, of pairs $(\bar{k}, \bar{\lambda})$ such that $\zeta(\bar{\lambda}) \leq \delta(\bar{k})$ but $\overline{\mathcal{H}(\bar{k}, \bar{\lambda})}$ is actually a proper subvariety of $\mathcal{G}$.

The following theorem shows that the weaker sufficient condition, stated above, for the existence of a parabolic (semi)stable bundle actually gives a necessary and sufficient condition for the existence of a parabolic (semi)stable bundle with a given parabolic data.

THEOREM 3.23. Let $(\bar{k}, \bar{\lambda}) \in K(n) \times \mathcal{I}(n)$ be such that $\overline{\mathcal{H}(\bar{k}, \bar{\lambda})}=\mathcal{G}$. If there is a parabolic semistable bundle, with the given parabolic data, then for every such pair $(\bar{k}, \bar{\lambda})$, the following inequality is valid:

$$
\omega(\bar{\lambda})-M n-\sum_{i=1}^{n} k_{i} \leq 0
$$

If furthermore there is a parabolic stable bundle, with the given parabolic data, then the left-hand side of the inequality (3.24) is strictly negative for every pair $(\bar{k}, \bar{\lambda})$ satisfying the above condition.

Proof. The first step will be to establish the following statement: if there is a parabolic semistable bundle with the given data, then there is one whose underlying vector bundle is $E$ defined in (3.12).

Let $W_{*}$ be a parabolic semistable bundle over $\mathbb{C P}^{1}$ of the given type, with $W$ as the underlying bundle.

There is a family of vector bundle $\mathcal{E} \longrightarrow \mathbb{C P}^{1} \times \mathcal{V}$ over $\mathbb{C P}^{1}$ parametrized by a vector space $\mathcal{V}$ such that for some $v \in \mathcal{V}$, the vector bundle $\left.\mathcal{E}\right|_{\mathbb{C P}^{1} \times v}$ is isomorphic to $W$, and over the general point of $\mathcal{V}$, the vector bundle is isomorphic to $E[\mathrm{Br}],[\mathrm{BH}]$. We quickly recall the construction of the family $\mathcal{E}$. Let $l$ be a sufficiently large integer such that both $E \otimes \mathcal{O}(l)$ and $W \otimes \mathcal{O}(l)$ are generated by global sections. Therefore, the vector bundle $V:=\mathcal{O}(-l)^{\oplus(r-1)}$ is a subbundle of both $E$ and $W$. Let $\mathcal{V}$ denote the vector space $H^{1}\left(\mathbb{C P}^{1}, \operatorname{Hom}(\mathcal{O}((r-1) l-M r+m), V)\right)$, parametrizing all extensions of the line bundle $\mathcal{O}((r-1) l-M r+m)$, which is isomorphic to $E / V$ or $W / V$, by the vector bundle $V$. So both $E$ and $W$ are represented in the universal family

$$
\mathcal{E} \longrightarrow \mathbb{C P}^{1} \times \mathcal{V}
$$

of extensions. In Proposition 3.18 we saw that the vector bundle $V(\bar{k})$ is rigid. The proof shows that $E$ is rigid. So there is a nonempty Zariski open subset of $\mathcal{V}$ over which the underlying vector bundle for the extension is isomorphic to $E$.

Let $U$ be a Zariski open subset of $\mathcal{V}$, containing a point corresponding to $W$, such that the restriction of $\mathcal{E}$ to any subvariety of the type $s \times U$, where $s \in S$, is algebraically trivializable. Actually $U$ can be taken to be the entire $\mathcal{V}$. Fixing trivializations of $\mathcal{E}$ over $s \times U$, the quasi-parabolic structure of $W_{*}$ is extended to all vector bundles parametrized by $U$. From the openness of the parabolic semistability condition, [Se], there is a nonempty Zariski open set $U^{\prime} \subseteq U$, such that for every $u \in U^{\prime}$, the corresponding parabolic bundle over $\mathbb{C P}^{1}$ is parabolic semistable.

Let $U^{\prime \prime}$ be the Zariski open dense subset of $\mathcal{V}$ consisting of all points for which the corresponding vector bundle is isomorphic to $E$. The property of this subset $U^{\prime \prime}$ that 
it is Zariski open and dense follows from the rigidity of $E$ established in the proof of Proposition 3.18.

Now for every $u \in U^{\prime} \cap U^{\prime \prime}$, the corresponding parabolic bundle is parabolic semistable and the underlying vector bundle is $E$.

The proof of the theorem will be completed by showing that if the inequality (3.24) fails then there cannot be any parabolic structure of the given type on $E$ which is semistable.

If $W_{*}$ is parabolic stable, then using the openness of the parabolic stability condition, and repeating the above argument, we get a parabolic stable bundle $E_{*}$ with $E$ as the underlying vector bundle.

Let $E_{*}$ be a parabolic semistable bundle of the given type, with $E$ as the underlying vector bundle. Take a $\bar{\lambda}=\prod_{s \in S} \lambda_{s} \in \mathcal{I}(n)$ and a $\bar{k}=\left\{k_{1}, \ldots, k_{n}\right\} \in K(n)$ such that $\overline{\mathcal{H}(\bar{k}, \bar{\lambda})}=\mathcal{G}$. We want to establish the inequality (3.24).

We have already remarked that $\mathcal{G}$ parametrizes the space of all quasi-parabolic structures on $E$. Let $U \subseteq \mathcal{G}$ be the nonempty Zariski open subset parametrizing the parabolic semistable structures. Let $E_{*}^{\prime}$ be a parabolic bundle corresponding to a point

$$
g \in U \cap \mathcal{H}(\bar{k}, \bar{\lambda})=U
$$

contained in the intersection of $U$ and $\mathcal{H}(\bar{k}, \bar{\lambda})$. Let $F \in \mathcal{M}(\bar{k})$ be a subbundle of $E$ such that

$$
g:=\prod_{s \in S} g_{s} \in \prod_{s \in S} S\left(F, \lambda_{s}\right)
$$

(in terms of the notation used in (3.15) and (3.16)).

Since $E_{*}^{\prime}$ is parabolic semistable, with $E$ as the underlying vector bundle, the parabolic degree of the subbundle $F$, with the parabolic structure induced by $E_{*}^{\prime}$ is nonpositive. The condition that $g_{s} \in S\left(F, \lambda_{s}\right)$ implies that the total parabolic weight of $F$ at the parabolic point $s$, for the parabolic structure induced by $E_{*}^{\prime}$, is at-least $\omega\left(s, \lambda_{s}\right)$, where the function $\omega(s,-)$ is defined in (3.6). As $\operatorname{deg} F=-M n-\sum_{i=1}^{n} k_{i}$, the inequality (3.26) is evidently a consequence of the condition that the parabolic degree of $F$ is nonpositive.

If $E_{*}$ is parabolic stable, then consider the parabolic structure on $E$ corresponding to a point in the intersection of $\mathcal{H}(\bar{k}, \bar{\lambda})$ and the Zariski open subset of $\mathcal{G}$ parametrizing parabolic stable structures on $E$. Repeating the above argument we immediately conclude that the left-hand side of (3.26) must be strictly negative. This completes the proof of Theorem 3.23.

If $r=2$ and $\# S=3$, and the parabolic weights at all the three parabolic points are $\{2 / 3,0\}$, then the inequalities in (3.11) are valid; but one of the strict inequalities in Theorem 3.23 is not satisfied (take $j=1$ ). Consider the direct sum of the trivial line bundle with the trivial parabolic structure and $\mathcal{O}(-2)$ with parabolic weights $2 / 3$ at each parabolic point. It is evidently a parabolic semistable bundle. However there is no parabolic stable bundle with this parabolic data at the parabolic points.

If $\# S$ is odd, $r=2$ and $\sum_{s \in S}\left(\alpha_{1}^{s}+\alpha_{2}^{S}\right)=1$, with at least one $\alpha_{2}^{s}$ being nonzero, then there is no parabolic semistable bundle with the parabolic data $\left\{\alpha_{1}^{s}, \alpha_{2}^{s}\right\}$. This is immediate after setting $j=0$ in the inequality in Theorem 3.23. 


\section{REFERENCES}

[BH] J. Brun And A. Hirschowitz, Droites de saut des fibrés stables de rang élevé sur $\mathbb{P}_{2}$, Math. Zeit., 181 (1982), pp. 171-178.

[Bi] I. BISWAS, A criterion for the existence of a parabolic stable bundle of rank two over the projective line, Int. Jour. Math., 9 (1998), pp. 523-533.

[Br] E. BRIESKorn, Über holomorphe $\mathbb{P}_{n}$-Bündel über $\mathbb{P}_{1}$, Math. Ann., 157 (1965), pp. 343-357.

[FS] M. FuRuta AND B. SteER, Seifert fibred homology 3-spheres and the Yang-Mills equations on Riemann surfaces with marked points, Adv. Math., 96 (1992), pp. 38-102.

[MS] V. Mehta And C. S. Seshadri, Moduli of vector bundles on curves with parabolic structures, Math. Ann., 248 (1980), pp. 205-239.

[N] N. Nitsure, Cohomology of the moduli of parabolic vector bundles, Proc. Ind. Acad. Sci. (Math. Sci.), 95 (1986), pp. 61-77.

[Se] C. S. Seshadri (RÉdigé par J.-M. Drezet), Fibrés Vectoriels Sur Les Courbes Algébriques, Astérisque, 96 (1982), Soc. Math. de France.

[Si1] C. Simpson, Harmonic bundles on noncompact curves, Jour. Amer. Math. Soc., 3 (1990), pp. 713-770.

[Si2] C. Simpson, Products of matrices, Canadian Mathematical Society Conference Proceedings, 12 (1992), pp. 157-185. 